University of Nebraska - Lincoln

DigitalCommons@University of Nebraska - Lincoln

April 1988

\title{
Magnetic properties, anisotropy, and microstructure of sputtered rare-earth iron multilayers
}

\author{
Z.S. Shan \\ University of Nebraska - Lincoln \\ S. Nafis \\ University of Nebraska - Lincoln \\ K.D. Aylesworth \\ University of Nebraska - Lincoln \\ David J. Sellmyer \\ University of Nebraska-Lincoln, dsellmyer@unl.edu
}

Follow this and additional works at: https://digitalcommons.unl.edu/physicssellmyer

Part of the Physics Commons

Shan, Z.S.; Nafis, S.; Aylesworth, K.D.; and Sellmyer, David J., "Magnetic properties, anisotropy, and microstructure of sputtered rare-earth iron multilayers" (1988). David Sellmyer Publications. 136. https://digitalcommons.unl.edu/physicssellmyer/136

This Article is brought to you for free and open access by the Research Papers in Physics and Astronomy at DigitalCommons@University of Nebraska - Lincoln. It has been accepted for inclusion in David Sellmyer Publications by an authorized administrator of DigitalCommons@University of Nebraska - Lincoln. 


\title{
Magnetic properties, anisotropy, and microstructure of sputtered rare-earth iron multilayers
}

\author{
Z. S. Shan, S. Natis, K. D. Aylesworth, and D. J. Sellmyer \\ Behlen Laboratory of Physics, University of Nebraska, Lincoln. Nebraska 68588-0111
}

\begin{abstract}
A study of compositionally modulated magnetic fims of the form Fe/RE, particularly for $R E=N d$ and $D y$, has been performed by vibrating sample magnetometry, ac susceptibility and $x$-ray diffraction. The reiationship between the magnetic properties and the layer thickness was studied systematicaily for $X-\AA \mathrm{Fe} / Y$ - $\AA$ Dy, as the layer thicknesses $X$ and $Y$ were varied from 1.8 to $20 \AA$. The ranges of layer thicknesses required for perpendicular anisotropy were determined. The interface and volume anisotropy energies were estimated for $X-\AA \mathrm{Fe} / \mathrm{Y}-\AA \mathrm{A} \mathrm{Nd}$ and the differences in the magnetic properties between $X \cdot \AA \mathrm{Fe} / 7-\AA \mathrm{A}$ y and $X-\AA \mathrm{Fe} / 7-\AA \mathrm{Nd}$ are discussed.
\end{abstract}

\section{INTRODUCTION}

Artificially structured magnetic multilayers are an interesting new class of materials. ${ }^{1}$ When the layer thicknesses approach the nanometer region there clearly is the opportunity to control the chenical short-range order and its anisotropy. Interfacial atomic interactions become increasingly significant and new thin-film properties, some with technological importance, are likely to arise. Previously we have studied $\mathrm{Fe} / \mathrm{Nd}$ (Ref. 2) and $\mathrm{Fe} / \mathrm{Ta}$ (Ref. 3) multilayers and have found that the single-ion anisotropy of the Nd ions plays a major role.

The purpose of this work is to investigate the range and the origins of perpendicular magnetic anisotropy in $\mathrm{Fe} / \mathrm{RE}$ multilayers. Five series of samples, $\mathrm{Fe} / \mathrm{Nd}, \mathrm{Co} / \mathrm{Nd}, \mathrm{Fe} / \mathrm{Dy}$, $\mathrm{Co} / \mathrm{Dy}$, and $\mathrm{Fe} / \mathrm{Er}$, have been prepared and measured. In this paper we shall rocus on some of the magnetic properties of $\mathrm{Fe} / \mathrm{Nd}$ and $\mathrm{Fe} / \mathrm{Dy}$ multilayer films only. A more detailed discussion of the other systems will be published elsewhere.

\section{EXPERIMENT}

$\mathrm{Fe} / \mathrm{RE}$ muitilayers were prepared in a multiple-gun sputtering system with a water-cooled rotating table. The substrate was mylar. The RE (Dy or Nd) was sputtered with an $f$ gun and the $F e$ with a de gun. The layer thickness was controlled by programming the time that the substrate was stationary above the corresponding target. The base pressure of the system was $2 \times 10^{-7}$ Forr and the pressure during sputtering was held at $5 \mathrm{~m}$ Torr in a continuous flow of highpurity argon gas. The total thickness of the samples was about $3000 \AA$. The multilayers were studied with $x$-ray diffraction, low- and high-field vibrating sample magnetometry, and ac susceptibility measurements.

\section{RESULTS AND DISCUSSION}

$X$-ray diffraction measurements have been made on selected multilayers and some examples are given in Ref. 2 . Briefly, the structure is polycrystalline for individual layer thickness greater than about $15 \AA$. For thinner layers the structure is amorphous in the direction normal to the film plane but the composition is modulated with the wavelength of the bilayer thickness.

The magnetization and anisotropy characteristics of $X$ -
$\AA \mathrm{Fe} / 7-\AA \mathrm{Nd}$ samples at $300 \mathrm{~K}$ are shown in Fig. 1. Several characteristics are noticed: (i) The films exhibit perpendicular anisotropy, i.e., $\sigma_{t}>\sigma_{\|}$, for $X=5 \AA, 7.5$, and $10 \AA$. The perpendicular anisotropy property gets stronger for $X$ ranging from 2.5 to $5 \AA$, then gets weaker as the $F e$ layer thickness increases and finally $\sigma_{\|}$becomes greater than $\sigma_{\perp}$ as $X$ becomes larger than $10 \AA$. We should point out that $5-\AA \mathrm{Fe} /$

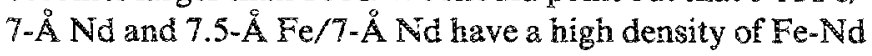
pairs oriented perpendicular to the films. (ii) $\mathrm{Fe} / \mathrm{Nd}$ multilayer films possess narrow hysteresis loops, and low values of coercivity and remanence, typically of the order of $100 \mathrm{Oe}$ and $1 \mathrm{emu} / \mathrm{g}$, respectively. (iii) Both of $\sigma_{1}$ and $\sigma_{\|}$increase as $X$ increases and more detailed features will be discussed later. The $X-\AA \mathrm{Fe} / 14 \AA \mathrm{A} \mathrm{Nd}$ and $X-\AA \mathrm{Fe} / 28-\AA$ Nd samples also have similar characteristics.

The temperature dependence of the hysteresis loops for 2.5- $\mathrm{Ae} / 10-\AA$ Nd is shown in Fig. 2. Of interest here is that

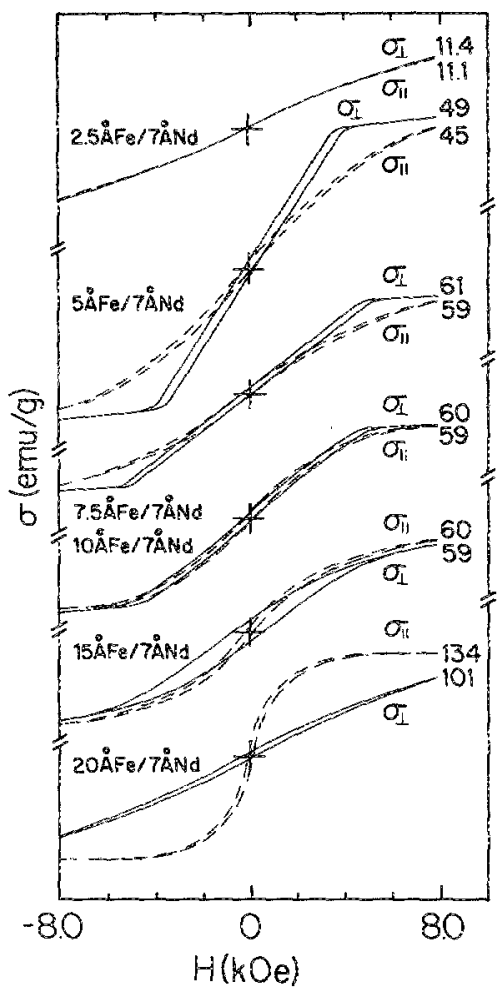

FIG. 1. Magnetization loops at $300 \mathrm{~K}$ for $X \cdot \AA \overline{\mathrm{Fe}} /$ 7-A Nd series. In this and subsequent figures the layer thicknesses are nominal values based on measurements of sputtering rate. 


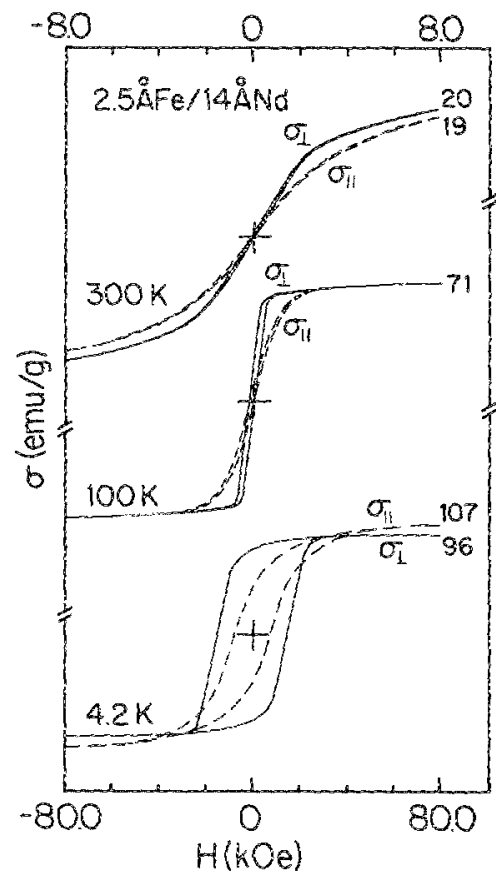

both the coercivity and the remanence increase rapidily as the temperature approaches $4.2 \mathrm{~K}$. We notice that $H_{c \mid l}=8 \mathrm{kOe}$, $H_{c l}=15 \mathrm{kOe}$, and $\sigma_{1} \approx \sigma_{1} \simeq 100 \mathrm{emu} / \mathrm{g}$ which is close to the calculated walue assurming that all $\mathrm{Fe}$ and $\mathrm{Nd}$ moments are aligned.

Using the formula ${ }^{4}$

$$
K_{u}=-\left[2 K_{i}+\left(K_{v}+2 \pi M_{0}^{2}\right) X\right] / \lambda_{q}
$$

where, $M_{0}, X, \lambda, K_{u}, K_{v}$, and $\mathbb{K}_{i}$ are saturation magnetiza. tion of $\mathrm{Fe}, \mathrm{Fe}$ layer thickness, bilayer thickness, and anisotropy energies corresponding to the bilayers, Fe layers, and interfaces, respectively. $K_{i}$ and $K_{v}$ can be determined from the experimental data. Figure 3 shows the $\lambda K_{t}$ vs $X$ curve. Several results are obtained: (i) The perpendicular magnetization occurs for $X<11 \AA$. (ii) From the $X=0$ intercept, we obtain $X_{i}=-0.18 \mathrm{erg} / \mathrm{cm}^{2}$. (iii) The bulk saturation magnetization data of $M_{0}=1714 \mathrm{emu} / \mathrm{cm}^{3}$ for Fe leads to $K_{y} \simeq-1.5 \times 10^{7} \mathrm{erg} / \mathrm{cm}$ and this is larger than the bulk value of $\mathrm{Fe}$ which is $4.8 \times 10^{5} \mathrm{erg} / \mathrm{cm}^{3}$. (iv) For $\mathrm{Fe}$ layer thickness less than $6-\AA \lambda K_{u}$ decreases as the Fe thickness

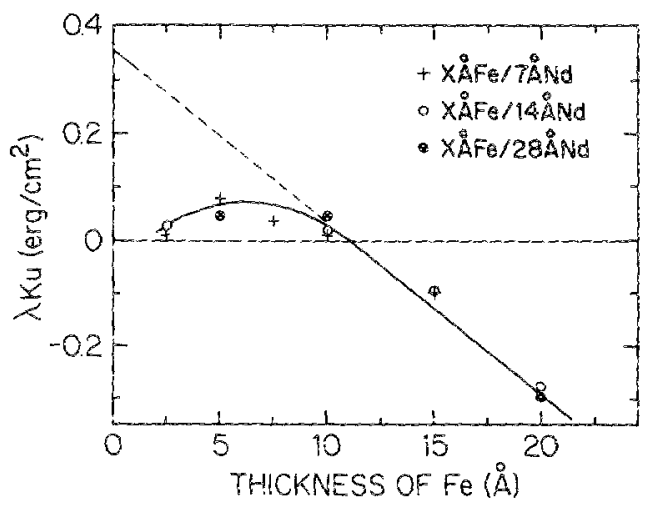

FIG. 3. Total anisotropy energy $K_{u}$ multiplied by the bilayer thickness vs the Fe layer thickness. decreases. This is because, as the Fe layer thickness becomes less than about 2 at. diam, significant mixing of the Nd into the Ee layer occurs. Thus, a wellodefned $\mathrm{Nd} / \mathrm{Fe}$ interface is lost.

In Fig. 4 the hysteresis loops for $X-\AA . A e / 3.5-\AA . D y$ as a function of the $\mathrm{Fe}$ layer thickness are shown. Several interesting features are found: (i) The samples of $X-\AA \mathrm{Fe} / 3.5-\AA$ Dy with $X=6,7.5,9$, and 12 A clearly possess perpendicu$12 r$ anisotropy. As the Fe layer thickness increases further the magnetization in the film plane becones dominant, i.e., $\sigma_{\| 1}>\sigma_{1}$. (ii) Comparing with Fig. 1 , the hysteresis loops with perpendicular anisotropy here are much broader; that is, these fims possess larger values of coereivity and remanence. The coercivity, remanence and the ratio of the remanence perpendicular to the film plane to that parallel to the fim plane are listed in Table I. It is seen clearly from these data that Fe/Dy multilayer film could be a potential candidate for a perpendicular recording material.

To determine the range of layer thickness regaired for perpendicular anisotropy, the Fe/Dy multiayer films were prepared systematically by increasing the layer thicknesses of Fe and Dy approximately one atomic layer at a time. Hysteresis loops were measured and some of the hysteresis loops are shown in Fig. 4. The results are summarized in Fig. 5. The rather broad region possessing perpendicular anisotropy also suggests that $\mathrm{Fe} / \mathrm{Dy}$ multilayers should be promising for perpendicular recording,

The magnetization for $X-\AA \mathrm{Fe} / 7 . \AA \mathrm{Nd}$ and $X-\AA \mathrm{Fe} / 7$. $\AA$ Dy samples as a function of Fe layer thickness $X$ are shown in Fig. 6. We notice the following. (i) The 8-kOe magnetizations $\sigma_{1}^{*}$ and $\sigma_{1}^{*}$ of $X-\AA \mathrm{Fe} / 7-\AA \mathrm{N}$ N are much larger than those of $X-\AA \mathrm{Fe} / 7 . \AA \mathrm{Dy}$ and this is associated with the character of the RE-TM monent coupling: Fe and Nd moments

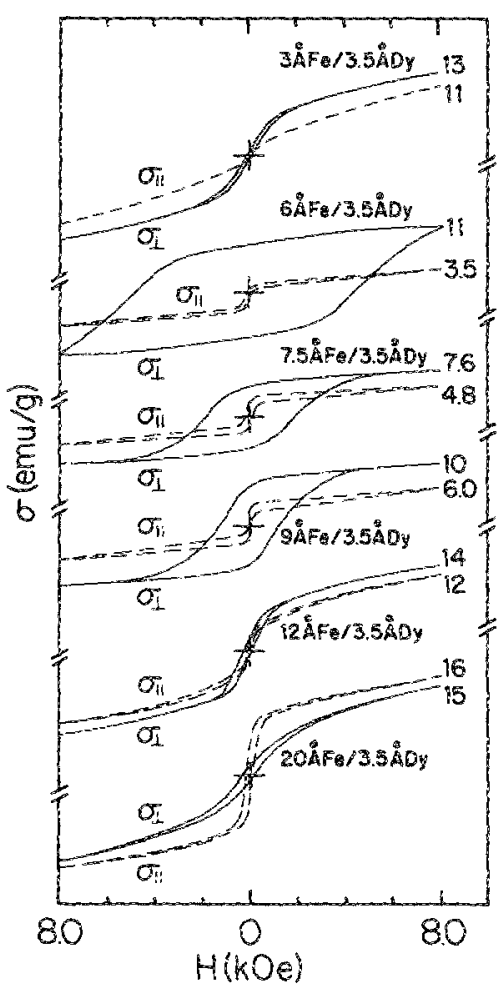

FIG. 4. Magnetization loops at $300 \mathrm{~K}$ for $X . \mathrm{Ae} /$ $3.5-\not 2$ Dy series. 
TABLE I. Coercivity and remanence of some $X-\AA$ Fe/3.5- $\AA$ Dy samples. $H_{i d}$ is the coercivity in the direction perpendicular to the film plane. $\sigma_{r j}$ and $\sigma_{\text {, }}$ are the magnetization at $h=0$ Oe in the direction perpendicular and parallel to the film plane, respectively.

\begin{tabular}{lccc} 
Samples & $\begin{array}{c}H_{c L} \\
(\mathrm{kOe})\end{array}$ & $\begin{array}{c}\sigma_{r i} \\
(\mathrm{emu} / \mathrm{g})\end{array}$ & $\sigma_{r i} / \sigma_{r+1}$ \\
\hline $6-\AA \mathrm{Fe} / 3.5-\AA \mathrm{Ay}$ & 5.2 & 7.0 & 12 \\
$7.5-\AA \mathrm{Fe} / 3.5-\AA \mathrm{Dy}$ & 1.9 & 4.9 & 2.6 \\
$9-\AA \mathrm{Fe} / 3.5-\AA \mathrm{Ay}$ & 1.2 & 6.5 & 4.3 \\
$12-\AA \mathrm{Fe} / 3.5-\AA \mathrm{Dy}$ & 0.18 & 1.5 & 0.95 \\
$20-\AA \mathrm{Fe} / 3.5-\AA \mathrm{Dy}$ & 0.20 & 1.0 & 0.22 \\
\hline
\end{tabular}

are parallel and for Fe and Dy they are antiparallel. (ii) For $X-\AA \mathrm{Fe} / 7 . \AA \mathrm{N}$ d the shape of the magnetization curve shows that as $X$ increases from 2.5 to $7.5 \AA$, $\sigma^{*}$ and $\sigma_{1}^{*}$ also increases presumably due to a decrease in the intermixing of the $\mathrm{Fe}$ and Nd layers so that the magnetic structure evolves from speromagnetic towards ferromagnetic. As $X$ increases from 7.5 to $16 \AA$, $\sigma^{*}$ and $\sigma_{1}^{*}$ remain constant, but a microscopic model for this behavior has yet to be developed. For $X \gg 16$ $\AA$, the magnetization increases rapidly and this is associated with the Fe layers becoming crystalline. (iii) It is of interest that there is a small depression of the magnetization curve at about $X=10 \sim 12 \AA$ for $X-\AA \mathrm{Fe} / 7 . \AA \mathrm{Dy}$. This may be associated with the moment directions of $F e$ and $D y$ which are expected to be antiparallel.

In summary, for $\mathrm{Fe} / \mathrm{Dy}$ and $\mathrm{Fe} / \mathrm{Nd}$ multilayers the perpendicular anisotropy and its dependence on the layer thicknesses of Fe or RE layer have been determined. The Fe/Dy multilayers, in particular, possess excellent magnetic prop-

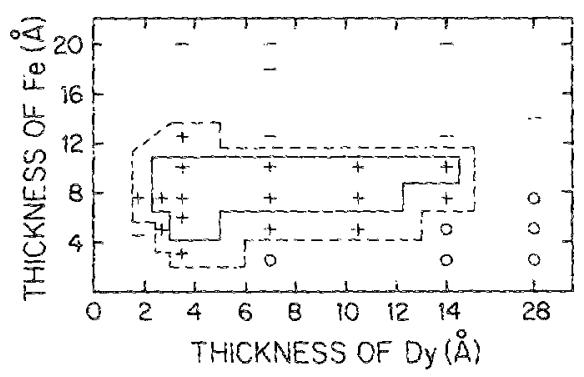

FIG. 5. The range of layer thicknesses exhibiting perpendicular or parallel anisotropy characteristics for $\mathrm{Fe} /$ Dy mulitlayer fims. + means $\sigma_{3}>\sigma_{\|}, \mathrm{O}$ means $\sigma_{1} \approx \sigma_{i}$, and - means $\sigma_{1}<\sigma_{11}$. The region surrounded by the solid line possesses $\left(\sigma_{r 1} / \sigma_{r \|}\right)>1.8$.

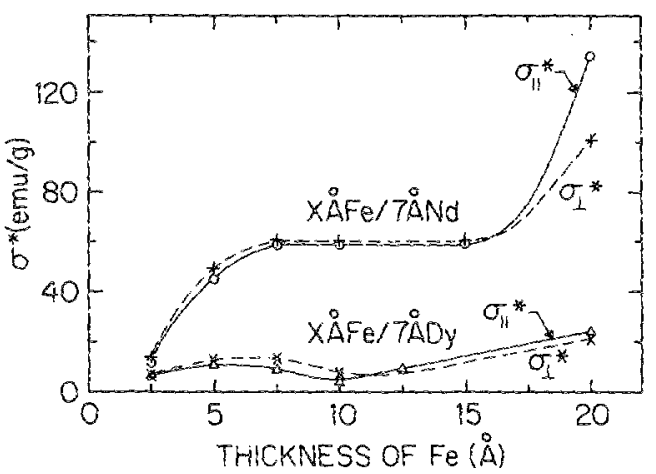

FIG. 6. Comparison of magnetization at $8 \mathrm{kOe}$ for $X$-Å Fe/7-A Nd and $X-\AA \mathrm{Fe} / 7-\mathrm{A} \mathrm{Dy}$.

erties in a rather broad region exhibiting strong perpendicular anisotropy and are suggested to be a potential candidate for perpendicular recording. The fact that the multilayers with higher density of $\mathrm{Fe}-\mathrm{Dy}$ ( or $\mathrm{Fe}-\mathrm{Nd}$ ) pairs oriented perpendicular to the films exhibits stronger perpendicular anisotropy suggests that such pairs are one of the important origins of the perpendicular anisotropy. However, further systematic studies will be required to determine the microscopic origin and other possible sources ${ }^{5}$ of the anisotropy. The TM-RE moment coupling for $\mathrm{Fe} / \mathrm{Nd}$ and $\mathrm{Fe} / \mathrm{Dy}$ thin multilayers has been observed clearly. The volume anisotropy energy $K_{v}$ found here is much larger than that of crystalline $\mathrm{Fe}$. One may speculate that this results from an amorphous or monocrystalline $\mathrm{Fe}$ structure of $X \leq 15 \AA$, but detailed microstructure studies will be required to test this hypothesis.

\section{ACKNOWLEDGRENTS}

We are grateful for financial support from the Solid State Physics Program of the National Science Foundation under Grant No. DMR-8605367 and to the Division of Intermal Program under Grant No. INT-8419546. Our thanks are due to $Z$. R. Zhao for helpful discussions and assistance.

'N. Sato and K. Habu, J. Appl. Phys. 61, 4287 (1987); F. J. A. den Broeder and H. C. Donkersloot, ibid. 61, 4317 (1987).

${ }^{2}$ D. I. Sellmyer, Z. R. Zhao, Z. S. Shan, and S. Nafis, J. Appl. Phys. 61, 4323 (1987).

${ }^{3}$ Z. S. Shan, Z. R. Zhao, J. G. Zhao, and D. I. Sellmyer, J. Appl. Phys. 61, 4320 (1987).

${ }^{4}$ P. F. Carcia, A. D. Meinhaldt, and A. Suna, Appi. Phys. Lett. 47, 178 (1987).

${ }^{5}$ A. Suna, J. Appl. Phys. 39, 313 (1986), and references therein. 